\title{
Mangiferin increases Nrf2 protein stability by inhibiting its ubiquitination and degradation in human HL60 myeloid leukemia cells
}

\author{
JIE ZHAO ${ }^{1}$, BENPING ZHANG ${ }^{1}$, SHANSHAN LI ${ }^{1}$, LINGLAN ZENG $^{2}$, YAN CHEN ${ }^{1}$ and JUN FANG ${ }^{1}$ \\ Departments of ${ }^{1}$ Hematology and ${ }^{2}$ Central Laboratory, Union Hospital, Tongji Medical College, \\ Huazhong University of Science and Technology, Wuhan 430022, P.R. China
}

Received December 2, 2013; Accepted February 25, 2014

DOI: $10.3892 /$ ijmm.2014.1696

\begin{abstract}
The nuclear factor erythroid 2-related factor 2 (Nrf2)-mediated antioxidant signaling pathway is a key target for cancer chemoprevention. Recent studies have that Nrf2 activation may be the result of an increase in Nrf2 protein stability. Mangiferin (MA), a compound monomer extracted from the mango plant, has antioxidant and cytoprotective activities. Our previous study demonstrated that MA increased Nrf2 expression and activated Nrf2 signaling in hematopoietic cells. Thus, in the present study, we aimed to investigate the mechanisms by which MA increases Nrf2 expression in human HL60 myeloid leukemia cells in vitro. Our western blot analysis results revealed that MA markedly increased Nrf2 expression in dose- and time-dependent manner. However treatment with MA did not affect the Nrf2 mRNA level. The results of cycloheximide (CHX)-chase analysis demonstrated that the Nrf2 protein half-life was prolonged to $58 \mathrm{~min}$ when the HL60 cells were pre-incubated with $50 \mu \mathrm{M}$ MA for $4 \mathrm{~h}$, whereas its half-life was only $20 \mathrm{~min}$ in the non-MA treated control cells. Further experiments revealed that MA mainly enhanced non-ubiquitinated Nrf2 protein levels when increasing Nrf2 protein stability; these effects differed from those induced by the proteasome inhibitor, MG132. Subsequent immunoprecipitation experiments confirmed that MA inhibited Nrf2 ubiquitination in HL60 cells. These results provide evidence that MA increases Nrf2 protein stability by inhibiting its ubiquitination and degradation in hematopoietic cells. This may be one of the mechanisms through which MA
\end{abstract}

Correspondence to: Dr Jun Fang or Professor Yan Chen, Department of Hematology, Union Hospital, Tongji Medical College, Huazhong University of Science and Technology, 1277 Jiefang Avenue, Wuhan 430022, P.R. China

E-mail: junfang@mail.hust.edu.cn

E-mail: yanchen@public.wh.hb.cn

Key words: mangiferin, nuclear factor erythroid 2-related factor 2, protein stabilization, ubiquitination, leukemia activates the Nrf2-mediated antioxidant response and exerts cytoprotective effects.

\section{Introduction}

The nuclear factor erythroid 2-related factor 2 (Nrf2)-mediated signaling pathway is a major cellular defense pathway against oxidative or electrophilic stress (1). Nrf2, a cap ' $n$ ' collar (CNC) basic leucine zipper transcription factor, can bind to antioxidant response element (ARE) in the nucleus, subsequently initiating the expression of genes encoding antioxidant and phase II drugmetabolizing enzymes (2-4). These genes include glutathione S-transferase (GST) $(5,6)$, NAD $(\mathrm{P}) \mathrm{H}$ : quinine reductases (NQO1) $(7,8)$ and heme oxygenase (HO-1) $(9,10)$, which contribute to detoxification and elimination of reactive oxidants and electrophilic agents. It has been documented that certain synthetic or natural compounds can activate the Nrf2-mediated signaling pathway and provide protection against environmental or chemical insults (11-14). The knockout of Nrf2 in mice has been shown to reduce not only basal, but also the inducible expression of antioxidant and phase II drug-metabolizing enzymes $(15,16)$. Silencing of the Nrf2 gene increases susceptibility to various oxidative stress-related pathologies, including chemical carcinogenesis, acetaminophen toxicity and hyperoxia (17-19). Therefore, Nrf2 has also been considered as a potential target for preventing inflammation-associated and chemical-induced carcinogenesis $(20,21)$.

However, the regulatory mechanisms involved in Nrf2 activation are not yet fully understood. Although it is well established that Nrf2 activity is controlled, at least in part, by the cytosolic protein, Kelch-like ECH-associated protein 1 (Keap1), the mechanisms by which Keap1 acts to repress Nrf2 activity remain to be fully characterized. Previously, it was considered that most of the Nrf2 proteins were bound to Keap1 and sequestered in cytoplasm under homeostatic conditions $(22,23)$. In response to oxidative stress or Nrf2 inducers/ activators, the Nrf2 protein can dissociate from Keap1, transfer from the cytoplasm to the nucleus, bind to ARE and lead to the subsequent transcription of ARE-regulated genes $(24,25)$. Thus, Nrf2 activation seems to be the result of its nuclear translocation. Nevertheless, a number of studies have suggested a different possible mechanism. Nrf2 was 
found to be primarily a nuclear protein. Under homeostatic conditions, Keap1 transiently shuttles the Nrf2 protein from the nucleus to the cytoplasm, prompts Nrf2 protein ubiquitination and degradation in the cytoplasm, maintains the intracellular Nrf2 protein at a basal level, thus repressing its activity (26). Oxidative stress or Nrf 2 inducers/activators regulate the interaction between Nrf2 and Keap1, inhibiting Nrf2 protein degradation and increasing its stability, which leads to Nrf2 protein accumulation in stressed cells. Therefore, Nrf2 activation has been suggested to be dependent on increasing Nrf2 protein stability $(1,14,27)$.

Mangiferin (MA), 2-C- $\beta$-D-glucopyranosyl-1,3,6,7-tetrahydroxyxanthone, is a compound monomer extracted from certain plants of the Anacardiaceae and Gentianaceae families, including Mangifera indica L. (mango), particularly in their leaves and bark (28). MA is widely used as a nutritional supplement, and as a cosmetic and phytomedicine in Southeast Asia and South America (29). This natural xanthone derivative has been reported to have various bioactivities, such as antioxidant, anti-tumor, anti-viral, anti-diabetic, anti-inflammatory, anti-allergic and immunomodulatory activities (28). Among these pharmacological activities, the antioxidant and cytoprotective properties of MA have been well elucidated. MA presents cardio-, hepato- and neuroprotective activities, as well as radioprotective activities against radiation $(14,28,29)$.

Previous stuides have revealed that MA induces Nrf2mediated antioxidant response, which provides an explanation for its antioxidant activity $(29,30)$. It has been reported that MA enhances the expression of several detoxification and antioxidant enzymes, including NQO1, GST, HO-1, superoxide dismutase (SOD) and uridin 5'-diphosphate-glucuronosyl transferase (UDP-GT) (33-36). Furthermore, MA has been shown to increase Nrf2 expression in D-galactosamine intoxicated rat liver (29). Moreover, in our previous study, we found that MA activated Nrf2-ARE signaling in human HL60 myeloid leukemia cells (30). However, to the best of our knowledge, the mechanisms by which MA increases Nrf2 expression have not been documented to date.

In this study, we demonstrate that MA increases $\mathrm{Nrf} 2$ expression, but not transcription in human HL60 myeloid leukemia cells. We provide evidence that MA prolongs the half-life of the Nrf2 protein by inhibiting its ubiquitination and degradation.

\section{Materials and methods}

Reagents. MA $\left(\mathrm{C}_{19} \mathrm{H}_{18} \mathrm{O}_{11}\right.$; molecular weight, 422.34) and cycloheximide (CHX, C15H23NO4; molecular weight, 281.4) were purchased from Sigma-Aldrich (St. Louis, MO, USA). MG132 $\left(\mathrm{C}_{26} \mathrm{H}_{42} \mathrm{~N}_{3} \mathrm{O}_{5}\right.$; molecular weight, 475.6) was obtained from Enzo Life Sciences (Farmingdale, NY, USA). MA and MG132 were initially dissolved in dimethyl sulfoxide (DMSO), stored at $-20^{\circ} \mathrm{C}$ and thawed on ice prior to use. $\mathrm{CHX}$ was dissolved in ultrapure water, stored at $-80^{\circ} \mathrm{C}$ and thawed on ice prior to use. RNAiso plus, PrimeScript RT Master Mix and SYBR Premix Ex Taq were purchased from Takara Bio, Inc. (Otsu, Japan). The primers used for real-time PCR were designed and synthesized by Takara Bio, Inc.. Rabbit polyclonal antibody against human Nrf2 (C-20) was obtained from Santa Cruz Biotechnology (Dallas, TX, USA). Mouse monoclonal antibody against human ubiquitin was obtained from Merck Millipore (Billerica, MA, USA). The BCA protein assay kit was from Pierce Biotechnology, Inc. (Rockford, IL, USA). The Image Lab enhanced chemiluminescence (ECL) detection system was from Bio-Rad (Hercules, CA, USA). Cell culture medium, RPMI-1640 and fetal abovine serum (FBS) were from HyClone (Logan, UT, USA).

Cell culture. The human HL60 myeloid leukemia cell line was kindly provided by Professor Jianfeng Zhou (Cancer Biology Research Center, Tongji Hospital, Wuhan, China), and then maintained in RPMI-1640 medium supplemented with $10 \%$ fetal bovine serum at $37^{\circ} \mathrm{C}$ in a humidified incubator containing $5 \% \mathrm{CO}_{2}$ in air.

Real-time PCR. Total RNA was isolated using RNAiso Plus according to the manufacturer's instructions. Total RNA was isolated using RNAiso Plus, and then $400 \mathrm{ng}$ total RNA were reverse transcripted into cDNA. Subsequently, $2 \mu \mathrm{l}$ cDNA were amplified with SYBR-Green Universal PCR Master mix in triplicate on a real-time PCR system (CFX96, Bio-Rad). Nrf2 mRNA levels related to $\beta$-actin levels were calculated using the $\Delta \mathrm{Ct}$ (cycle threshold) method. The primer sequences for human Nrf2 were: forward, 5'-ACTCCGGCATTTCAC TAAACACAAG-3' and reverse, 5'-CTGAGGCCAAGTAGT GTGTCTCCA-3'. The primer sequences for human $\beta$-actin were: forward, 5'-GCCCAGTCCTCTCCCAAGTC-3' and reverse, 5'-GGCACGAAGGTCATCATTC-3'. Control cells were processed in an identical manner apart from MA treatment.

Western blot analysis. For immunoblotting, whole-cell lysates were prepared using lysis buffer for $30 \mathrm{~min}$ on ice. Supernatants were collected as samples following centrifuged at 15,000 rpm for $10 \mathrm{~min}$ at $4^{\circ} \mathrm{C}$. Protein concentrations were determined using a BCA protein assay kit (Pierce Biotechnology, Inc.). Following denaturation, equal amounts of the protein extracts were resolved by $12 \%$ sodium dodecyl sulfate-polyacrylamide gel electrophoresis (SDS-PAGE) and transferred onto a polyvinylidene difluoride (PVDF) membrane. The membrane was blocked in phosphate-buffered saline (PBS) containing 5\% non-fat milk for $1 \mathrm{~h}$ at room temperature. It was subsequently incubated with anti-Nrf2 antibody (dilution 1:1,000) at $4^{\circ} \mathrm{C}$ overnight, followed by washing and treatment with HRP-labeled secondary antibody (Pierce Biotechnology, Inc.) for $2 \mathrm{~h}$ at room temperature. The blots were incubated with ECL reagent for $5 \mathrm{~min}$, and the signals were then detected with a chemiluminescence detection system (Bio-Rad). After stripping, the membrane was re-probed with human anti- $\beta$-actin antibody as a control for equal protein loading and protein integrity.

CHX-chase analysis. Nrf2 protein degradation was analyzed by $\mathrm{CHX}$-chase analysis. The HL60 cells were pre-incubated with or without $50 \mu \mathrm{M}$ MA for $4 \mathrm{~h}$. Subsequently, $100 \mu \mathrm{g} /$ $\mathrm{ml} \mathrm{CHX}$ were added to inhibit protein synthesis. The cells were collected at $0,5,15,30,45$ and 60 min after following treatment with $\mathrm{CHX}$, as previously described (31). Total protein $(60 \mu \mathrm{g})$ was fractionated on SDS-PAGE and blotted with anti-Nrf 2 and anti- $\beta$-actin antibodies. The results from western blot analysis were quantified by densitometry. Nrf2 protein half-lives were calculated from the slope of the semi- 
A

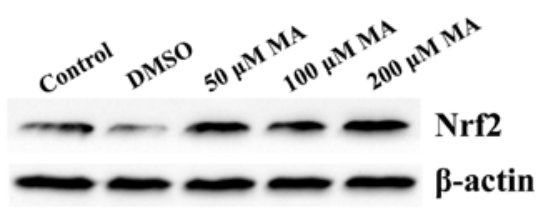

C

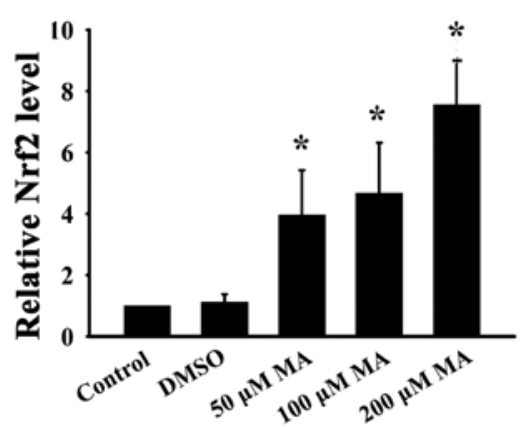

B

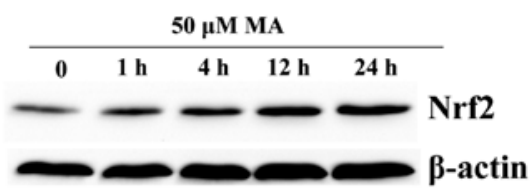

D

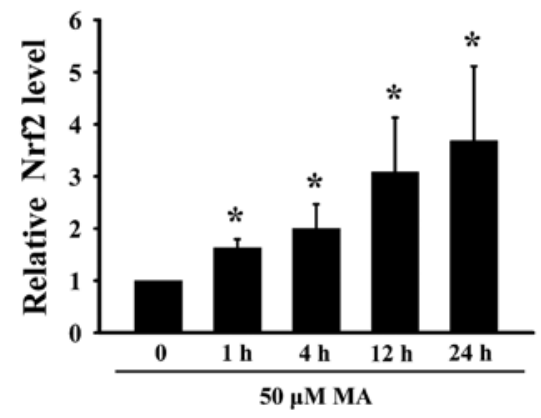

Figure 1. Mangiferin (MA) increases Nrf2 expression in HL60 cells. (A) Cells were treated with 0, 50, 100, 200 $\mu$ M MA and DMSO for 24 h. (B) Cells were treated with $50 \mu \mathrm{M}$ MA for $0,1,4,12,24 \mathrm{~h}$. Total cell lysates were subjected to western blot analysis with anti-Nrf 2 and anti- $\beta$-actin antibodies. $\beta$-actin was used as the control for equal protein loading and protein integrity. (C) The results in (A) were quantified by densitometry. (D) The results in (B) were quantified by densitometry. Nrf2 values were plotted after normalization to those of $\beta$-actin. Data represent the means \pm SD of 3 independent experiments $(* \mathrm{P}<0.05$, compared with the untreated control group).

logarithmically transformed best fit line. The decay curves were analyzed individually using linear regression of protein amount, and expressed as a percentage of protein remaining vs. time, as previously described (39).

Immunoprecipitation (IP). The HL60 cells were treated with $50 \mu \mathrm{M}$ MA, $10 \mu \mathrm{M}$ MG132, or a combination of $50 \mu \mathrm{M}$ MA and $10 \mu \mathrm{M}$ MG132 for $4 \mathrm{~h}$. The cells were washed twice with ice-cold PBS. The cells were then prepared in lysis/IP buffer [50 mM Tris (pH 8.0), $150 \mathrm{mM} \mathrm{NaCl}, 1 \%$ Nonidet P-40, $0.5 \%$ DOC, $0.1 \%$ SDS, $50 \mathrm{mM} \mathrm{NaF}, 1 \mathrm{mM} \mathrm{Na} \mathrm{VO}_{4}, 20 \mathrm{mM}$ $\beta$-glycerophosphate and $1 \mathrm{mM}$ okadaic acid] on ice for $30 \mathrm{~min}$, then centrifuged at $14,000 \mathrm{rpm}$ for $15 \mathrm{~min}$. Cell lysates were incubated with anti-Nrf 2 antibody at $4^{\circ} \mathrm{C}$ for $16 \mathrm{~h}$. The immune complexes were then precipitated with protein A-Sepharose beads at $4^{\circ} \mathrm{C}$ for an additional $2 \mathrm{~h}$. Subsequently, the precipitates were washed extensively with IP buffer, fractionated by SDS-PAGE and immunobloted with anti-ubiquitin antibody, as previously described (27). Control cells were processed in an identical manner apart from treatment with MA or/and MG132.

Statistical analysis. Data are expressed as the mean \pm SD of at least 3 independent experiments and processed by SPSS 17.0 statistical software for Windows. One-way ANOVA and Student-Newman-Keuls tests were applied for comparisons between each group. A value of $\mathrm{P}<0.05$ was considered to indicate a statistically significant difference.

\section{Results}

MA markedly increases Nrf2 expression in HL60 cells. To determine the effects of MA on Nrf2 expression, the HL60 cells were treated with MA in dose-and time-course experiments. The Nrf2 protein levels in whole cell lysate were then detected by western blot analysis. As shown in Fig. 1, the HL60 cells were treated with 50,100 or $200 \mu \mathrm{M}$ MA for $24 \mathrm{~h}$. These treatments resulted in a dose-dependent increase in the Nrf2 protein level. The Nrf2 protein level increased to 3.95-fold of the basal value following treatment with $50 \mu \mathrm{M} \mathrm{MA}$, and increased to nearly 7.56-fold of the basal level following treating with $200 \mu \mathrm{M}$ MA for $24 \mathrm{~h}$. In the time-course experiments, Nrf2 expression also markedly increased in a time-dependent manner when the HL60 cells were treated with $50 \mu \mathrm{M}$ MA for $1,4,12$ or $24 \mathrm{~h}$.

MA does not affect Nrf2 transcription in HL60 cells. To determine the effects of MA on Nrf2 transcription, the HL60 cells were treated with MA in dose-and time-course experiments as described above, and the Nrf2 mRNA levels were then detected by real-time PCR. As shown in Fig. 2, the Nrf2 mRNA levels did not differ significantly following treatment with MA, neither in a dose-dependent nor in a time-dependent manner.

MA prolongs the half-life of Nrf2 protein in HL60 cells. To determine the effects of MA on Nrf2 protein stability, the half-life of Nrf2 protein was calculated in the MA-treated and non-MA-treated HL60 cells. As shown in Fig. 3, the Nrf2 protein level decreased by $\sim 50 \%$ within $20 \mathrm{~min}$ in the non-MA-treated HL60 cells. Its half-life was only $20 \mathrm{~min}$. However, the Nrf2 protein level decreased by $\sim 50 \%$ after $50 \mathrm{~min}$ in the MA-treated cells. Its half-life was significantly prolonged to $58 \mathrm{~min}$. These data indicate that MA increases Nrf2 protein stability and prolongs its half-life.

MA increases Nrf2 stability by interfering with the ubiquitinproteasome protein degradation pathway. To investigate the mechanisms by which MA increases Nrf2 protein stability, the HL60 cells were treated with $50 \mu \mathrm{M}$ MA, $10 \mu \mathrm{M}$ of the 
A

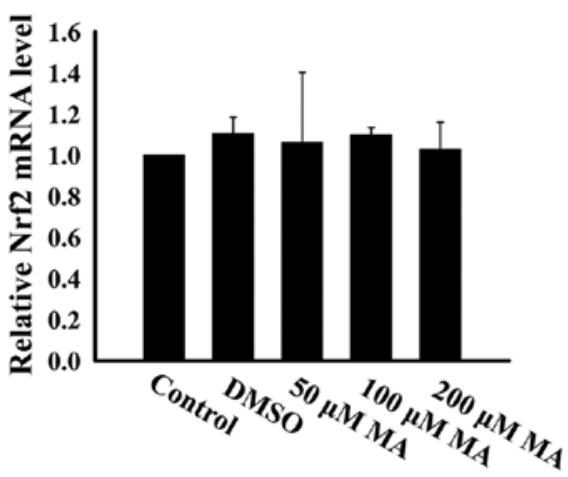

B

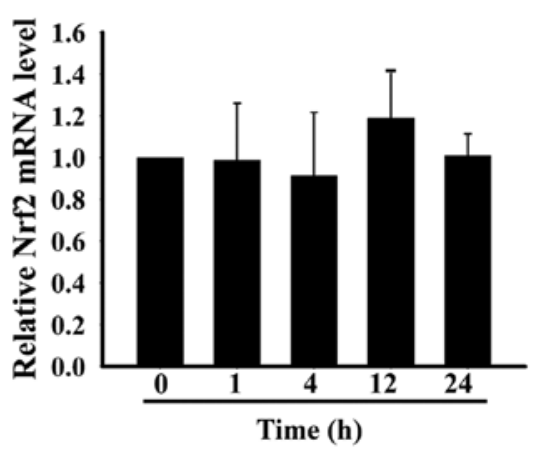

Figure 2. Mangiferin (MA) does not affect Nrf2 transcription in HL60 cells. (A) Cells were treated with 0, 50, 100, 200 $\mu$ M MA and DMSO for 24 h. (B) Cells were treated with $50 \mu \mathrm{M}$ MA for $0,1,4,12,24 \mathrm{~h}$. Total RNA was extracted and reverse-transcribed into cDNA, then cDNA was subjected to real-time PCR for the detection of Nrf 2 mRNA. Data represent the means \pm SD of 3 independent experiments.

A

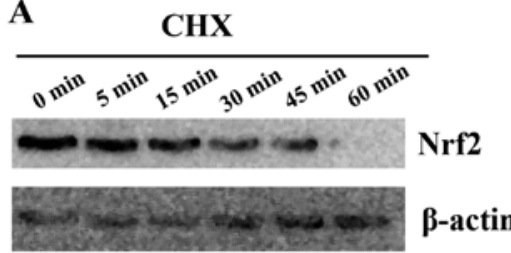

B

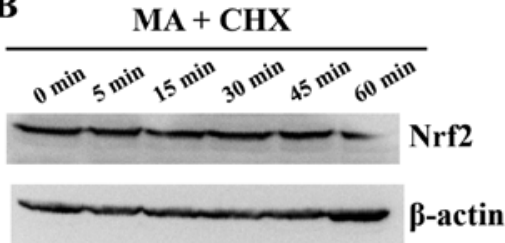

C

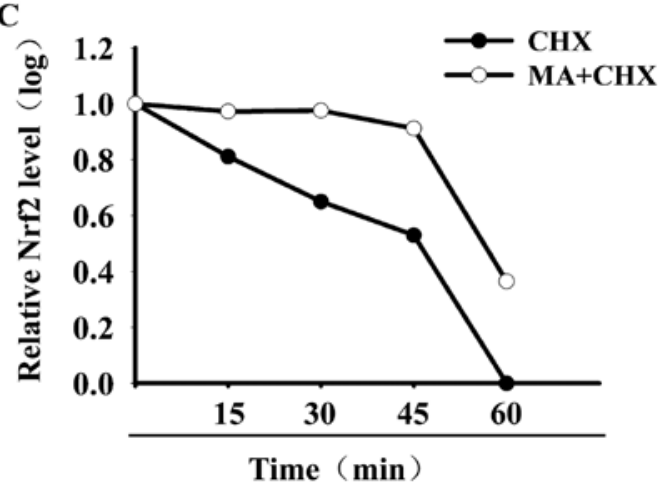

Figure 3. Mangiferin (MA) prolongs Nrf2 protein half-life in HL60 cells. (A) Cells were treated with $100 \mu \mathrm{g} / \mathrm{ml}$ cycloheximide (CHX) over a 1-h time period. (B) Cells were treated with $50 \mu \mathrm{M}$ MA for $4 \mathrm{~h}$ followed by $100 \mu \mathrm{g} / \mathrm{ml} \mathrm{CHX}$ over a $1 \mathrm{~h}$ time period. Cells were lysed at the indicated time points. Cell lysates were subjected to western blot analysis with anti-Nrf2 and anti- $\beta$-actin antibodies. $\beta$-actin was used as the control for equal protein loading and protein integrity. (C) The results in (A and B) were quantified by densitometry and plotted on a semi-log graph. The value of cells not treated with CHX was set as 1 . Data represent the means $\pm \mathrm{SD}$ of 3 independent experiments.

proteasome inhibitor, MG132, or a combination of $50 \mu \mathrm{M}$ MA and $10 \mu \mathrm{M}$ MG132 for $4 \mathrm{~h}$. The cells were then prepared for the analysis of the Nrf2 level by western blot analysis. The molecular weights of Nrf2 and poly-ubiquitinated Nrf2 are 57 and $100 \mathrm{kDa}$, respectively, according to the Nrf2 antibody data sheet (Santa Cruz Biotechnology). As shown in Fig. 4, the Nrf2/ poly-ubiquitinated Nrf2 protein level significantly increased to 1.88-, 3.81- and 3.82-fold of that of the control cells following treatment with MA, MG132 or the combination treatment, respectively. The total Nrf2 level following treatment with MG132 alone did not differ significantly from that observed following combined treatment with MA and MG132. Of note, MA mainly increased the non-ubiquitinated Nrf2 protein level. However, MG132 enhanced both the non-ubiquitinated and poly-ubiquitinated Nrf2 protein levels. These results indi- cate that MA increases Nrf2 protein stability by interfering with the ubiquitin-proteasome protein degradation pathway. However, the mechanisms involved differ from those of the proteasome inhibitor, MG132. MA inhibits ubiquitination, while MG132 suppresses proteasome activity.

MA inhibits Nrf2 ubiquitination in HL60 cells. To investigate whether MA suppresses Nrf2 ubiquitination, we performed IP experiments to pull down Nrf2 from the cell lysis of HL60 cells, and then identified ubiquitinated Nrf2 by immunoblot analysis with anti-ubiquitin monoclonal antibody. As shown in Fig. 5, the $10 \mathrm{kDa}$ band represents ubiquitinated Nrf2; the levels of ubiquitinated Nrf2 decreased when the HL60 cells were treated with MA. On the contrary, these conjugated proteins significantly accumulated when the cells were incu- 

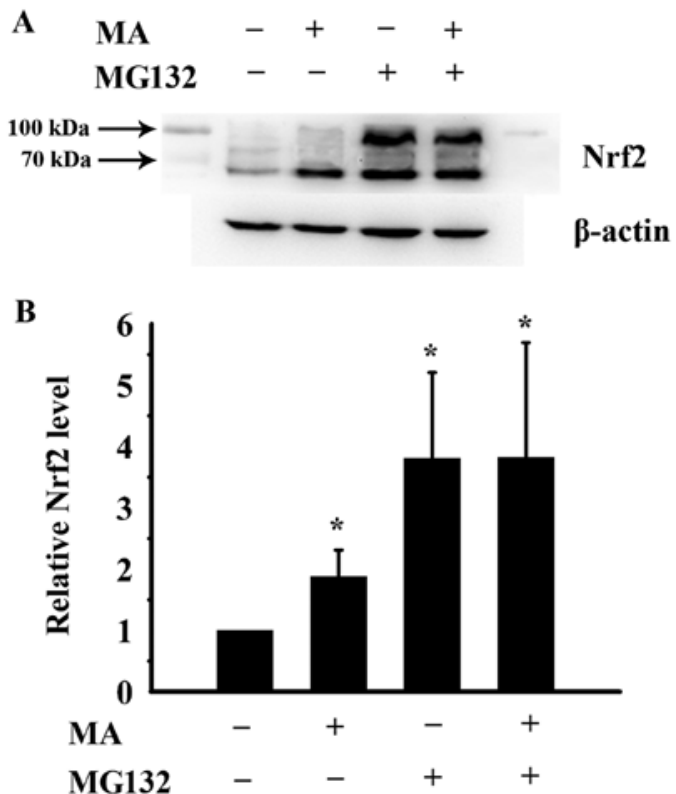

Figure 4. Mangiferin (MA) interferes with the ubiquitin-proteasome degradation of Nrf2 protein in HL60 cells. (A) Cells were treated with $50 \mu \mathrm{M}$ MA, $10 \mu \mathrm{M}$ MG132, or a combination of MA and MG132 for $4 \mathrm{~h}$. Total cell lysates were subjected to western blot analysis with anti-Nrf2 and anti- $\beta$-actin antibodies. $\beta$-actin was used as the control for equal protein loading and protein integrity. (B) The results in (A) were quantified by densitometry. Nrf2 values were plotted after normalization to those of $\beta$-actin. Data represent the means \pm SD of 3 independent experiments ( $\mathrm{P}<0.05$, compared with the non-treated control group).

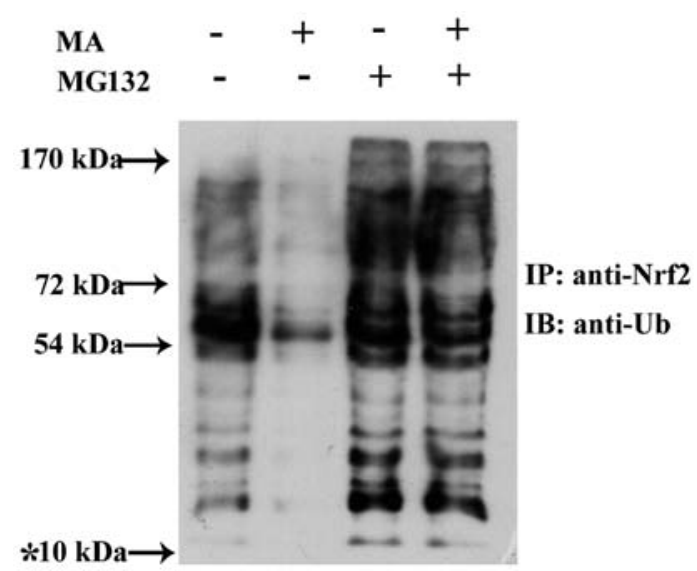

Figure 5. Mangiferin (MA) inhibits Nrf2 ubiquitination in HL60 cells. Cells were treated with $50 \mu \mathrm{M}$ MA, $10 \mu \mathrm{M}$ MG132, or a combination of MA and MG132 for $4 \mathrm{~h}$. Total cell lysates were immunoprecipitated with anti-Nrf2 antibody, and then immunoblotted with anti-ubiquitin antibody. ${ }^{~ R e p r e s e n t e d ~}$ ubiquitinated Nrf2.

bated with the proteasome inhibitor, MG132. These results suggest that MA decreases Nrf2 degradation by inhibiting its ubiquitination.

\section{Discussion}

The present study demonstrates that the natural antioxidant, mangiferin (MA), increases Nrf2 expression through posttranslational mechanisms in hematopoietic cells. MA enhances the Nrf2 protein level, but does not affect its transcription. MA reduces protein degradation and prolongs the half-life of $\mathrm{Nrf} 2$ by inhibiting its ubiquitination, which leads to its intracellular accumulation. These results provide evidence that MA enhances Nrf2 expression by interfering with the ubiquitinproteasome protein degradation pathway and increasing its protein stability. As mentioned above, MA activates Nrf2 and this activation may result from the increased protein stability and subsequent intracellular accumulation $(1,27)$. Therefore, our data suggest that MA activates the Nrf2-mediated signaling pathway by increasing Nrf2 stability.

Previous studies have confirmed that Nrf2 is targeted for rapid degradation by the ubiquitin-proteasome pathway. Nrf2 is a highly unstable protein and its half-life is only 15-30 min in unstressed cells (32-36). In our study, the Nrf2 half-life was $\sim 20 \mathrm{~min}$ in the HL60 cells and MA increased its half-life to $58 \mathrm{~min}$, which suggests that MA increases Nrf2 protein stability. Under homeostatic conditions, Nrf2 binds to its repressor, Keap1. Keap1 brings Nrf2 to the Cul3-E3 ubiquitin ligase and targets the $26 \mathrm{~S}$ proteasome for protein degradation (26). However, oxidative stress can antagonize the Keap1-Nrf2 interaction, increasing Nrf2 stability, leading to Nrf2 accumulation within cells $(32,33,35)$. Therefore, an increase in Nrf2 stability can be a result of the interference with the Keap1-Nrf2 interaction, inhibiting Nrf2 ubiquitination or reducing $26 \mathrm{~S}$ proteasome activity.

In the present study, MA markedly inhibited Nrf2 ubquitination, which may explain the increase in Nrf 2 stability. Certain natural or synthetic Nrf2 inducers/activators can also increase Nrf2 protein stability; however, the mechanisms involved are not completely similar to those of MA $(11-14,43)$. Treatment with tert-butylhydroquinone (tBHQ) has been shown to prolong the half-life of Nrf2 protein in human neural stem cells. However, tBHQ increases ubiquitinated Nrf2, bu MA decreases ubiquitinated Nrf2 (14). This Nrf2 activator seems to increase Nrf2 protein stability by stabilizing ubiquitinated Nrf2, which differs from MA. Oridonin, a diterpenoid purified from the Chinese medicinal herb, Rabdosia rubescens, was found to suppress Nrf2 ubiquitination and enhance Keap1 ubiquitination in human MDAMB-231 breast carcinoma cells (12). Thus, oridonin may induce a shift in ubiquitination from the substrate, Nrf2, to the substrate adaptor, Keap1. Ajoene, a stable garlic by-product, has been shown to inhibit the Nrf2-Keap1 interaction and decrease Nrf2 ubiquitination in HepG2 cells (13). The latter two Nrf2 activators increase Nrf2 stability through at least partly similar mechanisms to those of MA. Another Nrf2 activator 1, 2-dithiole-3-thione (D3T) has also been shown to significantly reduce the degradation of Nrf2 protein in PC12 cells. Nevertheless, it was unexplored as to which step of the ubiquitin-proteasome degradation of Nrf2 protein was affected by D3T (11). Therefore, Nrf2 activators can increase Nrf2 protein stability by interfering with the ubiquitin-proteasome pathway; however, the specific mechanisms involved may differ. The mechanisms through which MA inhibits Nrf2 ubiquitinationt require further investigation.

As a newly identified Nrf2 activator, MA may be a potential cytoprotective agent for hematopoietic cells, as well as a chemopreventive agent against leukemia. It has been well documented that Nrf2 activators/inducers exert cytoprotective effects through antioxidant mechanisms (37-39). Our 
previous study also demonstrated that MA relieved etoposide-induced DNA damage by activating Nrf2-mediated signaling and increasing NQO1 expression in mononuclear human umbilical cord blood (MNC hUCB) cells (Li S, et al, ASH Annual Meeting Abstracts 118: abs. 4626, 2011). This suggests that MA protects hematopoietic cells against injury induced by chemotherapy. Moreover, Nrf2 is a key target for chemoprevention against carcinogenesis (40-42). One major molecular mechanism is the induction of detoxification cytoprotective enzymes by Nrf2 activation (2). NQO1, a wellknown detoxification and cytoprotective enzyme, is subject to a genetic polymorphism (C609T) leading to the impairment of intermediate NQO1 activity. It has been reported that the NQO1 C609T polymorphism significantly increases the risk of treatment-related leukemia and myelodysplastic leukemia $(43,44)$. It has also been noted that oxidative stress is strongly associated with the relapse of acute myeloid leukemia and a poor prognosis $(45,46)$. Therefore, there is a possibility that MA may protect hematopoietic cells from leukemia genesis and relapse by activating the Nrf2-mediated antioxidant response; this, however, requires further investigation.

In conclusion, our study confirms that MA inhibits Nrf2 ubiquitination and increases its stability. This may be one of the mechanims through which it induces Nrf2 cellular accumulation and activates Nrf2-mediated signaling. MA may be a potential cytoprotective agent for hematopoietic cells and a chemopreventive agent against leukemia, which warrants further study.

\section{Acknowledgements}

This study was supported by grants from the National Natural Science Foundation of China (no. 30900632 and no. 81372541). The authors would like to thank the Department of Central Laboratory, Union Hospital, Tongji Medical College, Huazhong University of Science and Technology, Wuhan, China, for providing relevant experimental facilities and technical support.

\section{References}

1. Nguyen T, Nioi P and Pickett CB: The Nrf2-antioxidant response element signaling pathway and its activation by oxidative stress. J Biol Chem 284: 13291-13295, 2009.

2. Eggler AL, Gay KA and Mesecar AD: Molecular mechanisms of natural products in chemoprevention: induction of cytoprotective enzymes by Nrf2. Mol Nutr Food Res 52 (Suppl 1): S84-S94, 2008.

3. Huang HC, Nguyen $\mathrm{T}$ and Pickett CB: Regulation of the antioxidant response element by protein kinase $\mathrm{C}$-mediated phosphorylation of NF-E2-related factor 2. Proc Natl Acad Sci USA 97: 12475-12480, 2000.

4. Nguyen T, Huang HC and Pickett CB: Transcriptional regulation of the antioxidant response element. Activation by Nrf2 and repression by MafK. J Biol Chem 275: 15466-15473, 2000.

5. Friling RS, Bensimon A, Tichauer Y, et al: Xenobiotic-inducible expression of murine glutathione S-transferase Ya subunit gene is controlled by an electrophile-responsive element. Proc Natl Acad Sci USA 87: 6258-6262, 1990.

6. Casalino E, Calzaretti G, Landriscina M, et al: The Nrf2 transcription factor contributes to the induction of alpha-class GST isoenzymes in liver of acute cadmium or manganese intoxicated rats: comparison with the toxic effect on $\mathrm{NAD}(\mathrm{P}) \mathrm{H}$ :quinone reductase. Toxicology 237: 24-34, 2007.
7. Liang L, Gao C, Luo M, et al: Dihydroquercetin (DHQ) induced HO-1 and NQO1 expression against oxidative stress through the Nrf2-dependent antioxidant pathway. J Agric Food Chem 61: 2755-2761, 2013.

8. Kim HJ, Zheng M, Kim SK, et al: CO/HO-1 induces NQO-1 expression via Nrf2 activation. Immune Netw 11: 376-382, 2011.

9. Lee YM, Auh QS, Lee DW, et al: Involvement of nrf2-mediated upregulation of heme oxygenase-1 in mollugin-induced growth inhibition and apoptosis in human oral cancer cells. Biomed Res Int 2013: 210604, 2013.

10. Maruyama A, Mimura J, Harada N, et al: Nrf2 activation is associated with Z-DNA formation in the human HO-1 promoter. Nucleic Acids Res 41: 5223-5234, 2013.

11. Dong J, Yan D and Chen SY: Stabilization of Nrf2 protein by D3T provides protection against ethanol-induced apoptosis in PC12 cells. PLoS One 6: e16845, 2011.

12. Du Y, Villeneuve NF, Wang XJ, et al: Oridonin confers protection against arsenic-induced toxicity through activation of the Nrf2-mediated defensive response. Environ Health Perspect 116: 1154-1161, 2008.

13. Kay HY, Won Yang J, Kim TH, et al: Ajoene, a stable garlic by-product, has an antioxidant effect through Nrf2-mediated glutamate-cysteine ligase induction in HepG2 cells and primary hepatocytes. J Nutr 140: 1211-1219, 2010.

14. Li J: Stabilization of Nrf2 by tBHQ confers protection against oxidative stress-induced cell death in human neural stem cells. Toxicol Sci 83: 313-328, 2004.

15. Kurzatkowski DM and Trombetta LD: Maneb causes pro-oxidant effects in the hippocampus of Nrf2 knockout mice. Environ Toxicol Pharmacol 36: 427-436, 2013.

16. Mukhopadhyay S, Sekhar KR, Hale AB, et al: Loss of NRF2 impairs gastric nitrergic stimulation and function. Free Radic Biol Med 51: 619-625, 2011.

17. Yates MS, Tran QT, Dolan PM, et al: Genetic versus chemoprotective activation of Nrf2 signaling: overlapping yet distinct gene expression profiles between Keap1 knockout and triterpenoid-treated mice. Carcinogenesis 30: 1024-1031, 2009.

18. Ni HM, Boggess N, McGill MR, et al: Liver-specific loss of Atg 5 causes persistent activation of Nrf2 and protects against acetaminophen-induced liver injury. Toxicol Sci 127: 438-450, 2012.

19. Reddy NM, Kleeberger SR, Kensler TW, et al: Disruption of Nrf2 impairs the resolution of hyperoxia-induced acute lung injury and inflammation in mice. J Immunol 182: 7264-7271, 2009.

20. Kundu JK and Surh YJ: Nrf2-Keap1 signaling as a potential target for chemoprevention of inflammation-associated carcinogenesis. Pharm Res 27: 999-1013, 2010.

21. Zhao CR, Gao ZH and Qu XJ: Nrf2-ARE signaling pathway and natural products for cancer chemoprevention. Cancer Epidemiol 34: 523-533, 2010.

22. Tong KI, Kobayashi A, Katsuoka F, et al: Two-site substrate recognition model for the Keap1-Nrf2 system: a hinge and latch mechanism. Biol Chem 387: 1311-1320, 2006.

23. McMahon M, Thomas N, Itoh K, et al: Dimerization of substrate adaptors can facilitate cullin-mediated ubiquitylation of proteins by a 'tethering' mechanism: a two-site interaction model for the Nrf2-Keap1 complex. J Biol Chem 281: 24756-24768, 2006.

24. Dhakshinamoorthy S and Jaiswal AK: Functional characterization and role of INrf2 in antioxidant response element-mediated expression and antioxidant induction of NAD $(\mathrm{P}) \mathrm{H}:$ quinone oxidoreductase1 gene. Oncogene 20: 3906-3917, 2001.

25. Malhotra D, Portales-Casamar E, Singh A, et al: Global mapping of binding sites for Nrf2 identifies novel targets in cell survival response through ChIP-Seq profiling and network analysis. Nucleic Acids Res 38: 5718-5734, 2010.

26. Nguyen T: Nrf2 controls constitutive and inducible expression of ARE-driven genes through a dynamic pathway involving nucleocytoplasmic shuttling by Keap1. J Biol Chem 280: 32485-32492, 2005.

27. Nguyen T: Increased protein stability as a mechanism that enhances Nrf2-mediated transcriptional activation of the antioxidant response element. Degradation of Nrf2 by the $26 \mathrm{~S}$ proteasome. J Biol Chem 278: 4536-4541, 2002.

28. Luo F, Lv Q, Zhao Y, et al: Quantification and purification of mangiferin from Chinese mango (Mangifera indica L.) cultivars and its protective effect on human umbilical vein endothelial cells under $\mathrm{H}_{2} \mathrm{O}_{2}$-induced stress. Int J Mol Sci 13: 11260-11274, 2012. 
29. Das J, Ghosh J, Roy A and Sil PC: Mangiferin exerts hepatoprotective activity against D-galactosamine induced acute toxicity and oxidative/nitrosative stress via Nrf2-NFкB pathways. Toxicol Appl Pharmacol 260: 35-47, 2012.

30. Zhang BP, Zhao J, Li SS, et al: Mangiferin activates Nrf2antioxidant response element signaling without reducing the sensitivity to etoposide of human myeloid leukemia cells in vitro. Acta Pharmacol Sin 35: 257-266, 2014.

31. He X and Ma Q: NRF2 cysteine residues are critical for oxidant/ electrophile-sensing, Kelch-like ECH-associated protein1-dependent ubiquitination-proteasomal degradation, and transcription activation. Mol Pharmacol 76: 1265-1278, 2009.

32. Stewart D, Killeen E, Naquin R, et al: Degradation of transcription factor Nrf2 via the ubiquitin-proteasome pathway and stabilization by cadmium. J Biol Chem 278: 2396-2402, 2003.

33. Gu B, Johnston VK, Gutshall LL, et al: Arresting initiation of hepatitis $\mathrm{C}$ virus RNA synthesis using heterocyclic derivatives. J Biol Chem 278: 16602-16607, 2003

34. McMahon M, Itoh K, Yamamoto M, et al: Keap1-dependent proteasomal degradation of transcription factor Nrf2 contributes to the negative regulation of antioxidant response element-driven gene expression. J Biol Chem 278: 21592-21600, 2003.

35. Itoh K, Wakabayashi N, Katoh Y, et al: Keap1 regulates both cytoplasmic-nuclear shuttling and degradation of Nrf2 in response to electrophiles. Genes Cells 8: 379-391, 2003.

36. Zhang DD and Hannink M: Distinct cysteine residues in Keap1 are required for Keap1-dependent ubiquitination of $\mathrm{Nrf} 2$ and for stabilization of Nrf2 by chemopreventive agents and oxidative stress. Mol Cell Biol 23: 8137-8151, 2003.

37. Shehzad A and Lee YS: Molecular mechanisms of curcumin action: signal transduction. Biofactors 39: 27-36, 2013.
38. Dinkova-Kostova AT: Chemoprotection against cancer by isothiocyanates: a focus on the animal models and the protective mechanisms. Top Curr Chem 329: 179-201, 2013.

39. Wang S, Penchala S, Prabhu S, et al: Molecular basis of traditional Chinese medicine in cancer chemoprevention. Curr Drug Discov Technol 7: 67-75, 2010.

40. Hayes JD, McMahon M, Chowdhry S, et al: Cancer chemoprevention mechanisms mediated through the Keap1-Nrf2 pathway. Antioxid Redox Signal 13: 1713-1748, 2010.

41. Hybertson BM, Gao B, Bose SK, et al: Oxidative stress in health and disease: the therapeutic potential of Nrf2 activation. Mol Aspects Med 32: 234-246, 2011.

42. Slocum SL and Kensler TW: Nrf2: control of sensitivity to carcinogens. Arch Toxicol 85: 273-284, 2011.

43. Naoe T, Takeyama K, Yokozawa T, et al: Analysis of genetic polymorphism in NQO1, GST-M1, GST-T1, and CYP3A4 in 469 Japanese patients with therapy-related leukemia/myelodysplastic syndrome and de novo acute myeloid leukemia. Clin Cancer Res 6: 4091-4095, 2000.

44. Larson RA, Wang Y, Banerjee M, et al: Prevalence of the inactivating $609 \mathrm{C}-->\mathrm{T}$ polymorphism in the $\mathrm{NAD}(\mathrm{P}) \mathrm{H}:$ quinone oxidoreductase (NQO1) gene in patients with primary and therapy-related myeloid leukemia. Blood 94: 803-807, 1999.

45. Li W and Kong AN: Molecular mechanisms of Nrf2-mediated antioxidant response. Mol Carcinog 48: 91-104, 2009.

46. Zhou FL, Zhang WG, Wei YC, et al: Involvement of oxidative stress in the relapse of acute myeloid leukemia. J Biol Chem 285: 15010-15015, 2010. 